\title{
Prediction of Binding Sites in Receptor-Ligand Complexes with the Gaussian Network Model
}

\author{
Turkan Haliloglu, ${ }^{1}$ Emek Seyrek, ${ }^{1}$ and Burak Erman ${ }^{2}$ \\ ${ }^{1}$ Polymer Research Center, Bogazici University, Bebek, Istanbul, Turkey \\ ${ }^{2}$ Department of Chemical and Biological Engineering, Koc University, Sariyer, 34450 Istanbul, Turkey
}

(Received 7 September 2007; published 3 June 2008)

Residues at the binding sites of the ligand and receptor of several enzyme-inhibitor and antibodyantigen complexes are predicted from the slowest (for the ligand) and fastest (for the receptor) modes of motion by the Gaussian Network Model applied to unbound molecules.

Protein-protein binding prediction is defined as estimating the binding sites of both monomers of the complex structures in their unbound states. The complex structures reveal that the interfaces are not mostly flat and smooth but rather have some residue side chains protruding into the cavities in the other protein [1,2], and there is a small number of residues at the interface of the complex structure, namely, hotspots, which account for most of the binding energy [3]. With the question of whether the architecture of a binding site is already imprinted in the unbound state of the protein or not, several studies have implications that binding is related to the local characteristics of the unbound protein [4]. The local mechanism of protein-protein recognition has recently been elucidated in terms of anchor side chains that provide a geometrical fit that is based on lock-and-key and induced-fit processes [1]. Both of these processes depend on the adoption of the correct orientation and position of the two proteins.

In this Letter, we study the binding of non-obligate enzyme-inhibitor and antibody-antigen complexes which belong to the class of mostly permanent interfaces and transient interfaces of intermediate strength [5]. Using the Gaussian Network Model (GNM) [6,7], we show that residues involved in binding can be predicted without direct reference to geometrical matching and local properties. We also discuss the applicability of the proposed model for heterodimers of comparable sizes and homodimers, and higher oligomers. The proposed mechanism identifies the binding sites of both the ligand (the smaller protein) and the receptor (the larger protein), based on the collective vibrational dynamics of each, obtained in the unbound state. The search of a ligand for a binding partner is a highly nonlocal dynamic process in which all residues are collectively involved. We also show that the ligand and receptor identify their binding sites through their slowest and fastest vibrational modes, respectively. Complementing the latter, we show that an external harmonic force couples the slowest mode fluctuations of the anchor residue to the rest of the ligand, and the fastest mode fluctuations of the binding residue at the receptor to the rest of the receptor.

We studied 15 ezyme-inhibitor and antibody-antigen complex structures for which the anchor residues have been determined by molecular dynamics calculations by Camacho and colleauges [1]. The corresponding residues on the receptor, i.e., the closest contact residues of the anchor residues are the anchoring groove residues [1]. Our calculations show that the anchor residues exhibit large scale fluctuations that are strongly anticorrelated, in the slowest mode, with the remaining residues of the ligand. This gives the anchor residue the ability to probe its environment. There is a large anticorrelation between the anchor residue and the terminal residues at the tails of the ligand. The binding groove sites on the receptor at which the anchor residue will bind are located in the most constrained regions of the protein, and this site is reflected by the fast frequency modes of fluctuations [8,9]. Although intuitively straightforward and simple, the collective fluctuation dynamics presented here predicts the residues responsible in binding for both the ligand and receptor. The systems studied are shown in Table I. The second column indicates the residue obtained by the slowest mode of the ligand. This residue is the one that displays the highest relative fluctuations in that anchor loop. The residues obtained by Camacho [1] are presented in square brackets. Numbers in parentheses show the evolutionary conservation level calculated using the ConSeq web server [11]. The conservation scores reflect the bayesian measure of the site's evolutionary rate. The positions that evolve slowly and rapidy are commonly referred to as "conserved" and "variable," respectively. The continuous conservation scores were partitioned into a discrete scale of 9 bins. A grade of 1 indicates a highly variable amino acid position, and 9 is highly conserved.

Model. - Our predictions are based on the Gaussian network Model (GNM) [6] with the Hamiltonian $H=$ $\frac{1}{2} \Delta \boldsymbol{R}^{T} \boldsymbol{\Gamma} \Delta \boldsymbol{R}$ where $\Delta \boldsymbol{R}$ is the column vector of fluctuations of residues, and $\boldsymbol{\Gamma}$ is given as

$$
\boldsymbol{\Gamma}_{i j}=\left\{\begin{array}{lll}
-\gamma^{*} & i \neq j \text { and } & R_{i j} \leq r_{\text {cutoff }} \\
0 & i \neq j \text { and } & R_{i j}>r_{\text {cutoff }} \\
-\sum_{k} \gamma^{*} & i=j \neq k
\end{array}\right.
$$

Here, $R_{i j}$ is the distance between residue $i$ and $j, r_{\text {cutoff }}$ is the distance that defines the neighborhood condition, generally taken between $6.5-7 \AA$, and $\gamma^{*}$ is a scaling parame- 
TABLE I. List of enzyme-inhibitor and antibody-antigen complex [10]. Numbers in square brackets are results of Camacho; numbers in parentheses are conservation levels.

\begin{tabular}{|c|c|c|c|}
\hline PDB ID & $\begin{array}{l}\text { Slowest mode residue } \\
\text { (Ligand) }\end{array}$ & $\begin{array}{l}\text { Fastest mode residue } \\
\text { (Receptor) }\end{array}$ & $\begin{array}{c}\text { Distance }(\AA) \\
\text { (between closest atoms) }\end{array}$ \\
\hline \multicolumn{4}{|c|}{ Antigen-Antibody } \\
\hline $1 \mathrm{MEL}$ & ASN59 (9) [TRP62 (1)] & ALA104 (7) & 2.7 \\
\hline $1 \mathrm{JHL}$ & ASN59 (9) [LYS116(5)] & ASP99 (1) & 3.0 \\
\hline $1 \mathrm{BQL}$ & ASP48 (6) [ARG45 (2)] & TYR33 (1) & 5.9 \\
\hline $1 \mathrm{BVK}$ & GLY117 (7) [GLN121(1)] & ILE51 (8) & 6.8 \\
\hline \multicolumn{4}{|c|}{ Enzyme-Inhibitor } \\
\hline $1 \mathrm{ACB}$ & LEU45(1)] [Leu45(1)] & ASP194 (9) & 3.3 \\
\hline $1 \mathrm{BRC}$ & ARG15 (4) [Arg15(4)] & ASP194 (9), GLY197 (9) & 3.1 \\
\hline $1 \mathrm{BRS}^{\mathrm{a}}$ & ASP39 [Asp39] & SER85 & 6.7 \\
\hline $1 \mathrm{CHO}$ & LEU18 (1) [Leu18(1)] & ASP194 (9) & 3.4 \\
\hline $1 \mathrm{CSE}$ & LEU45(1) [Leu45(1)] & VAL177 (9) & 7.0 \\
\hline 1TGS & LYS18 (3) [Lys18(3)] & ASP194 (9) & 3.4 \\
\hline $1 \mathrm{UGH}^{\mathrm{a}}$ & MET24 & VAL 274(9) [LEU272] & 6.9 \\
\hline 2PTC & LYS15 (4) [LYS15(4)] & ASP 194(9), GLY197 (9) & 3.1 \\
\hline 2 SIC & MET70 (1) [MET70(1)] & VAL95 (8) & 6.6 \\
\hline 2SNI & ILE56 (4) [ILE56(4)] & VAL95 (8) & 6.4 \\
\hline 2TEC & LYS15 (4) [LEU45(1)] & THR224 (9) & 3.5 \\
\hline
\end{tabular}

${ }^{\mathrm{a}}$ Not enough sequences for conservation analysis [11].

ter. The correlation $\left\langle\Delta \boldsymbol{R}_{i} \cdot \Delta \boldsymbol{R} j\right\rangle$ between the fluctuations of residue $i$ and $j$ is then obtained according to the known operations as

$$
\left\langle\Delta \boldsymbol{R}_{i} \cdot \Delta \boldsymbol{R}_{j}\right\rangle=\frac{3}{2}\left(\boldsymbol{\Gamma}^{-1}\right)_{i j}=\frac{3}{2} \sum_{k} \lambda_{k}^{-1}\left(\boldsymbol{u}_{k}\right)_{i}\left(\boldsymbol{u}_{k}\right)_{j}
$$

where, $\lambda_{k}$ and $\mathbf{u}_{k}$ are the kth nonzero eigenvalue and corresponding normalized eigenvector of the $\Gamma$ matrix.

Relative fluctuations $\left\langle\Delta R_{i j}^{2}\right\rangle \equiv\left\langle\left(\Delta \boldsymbol{R}_{i}-\Delta \boldsymbol{R}_{j}\right)^{2}\right\rangle$ of residues $i$ and $j$ are defined as

$$
\begin{aligned}
\left\langle\Delta R_{i j}^{2}\right\rangle & =\left\langle\Delta R_{i}^{2}\right\rangle-2\left\langle\Delta \boldsymbol{R}_{i} \cdot \Delta \boldsymbol{R}_{j}\right\rangle+\left\langle\Delta R_{j}^{2}\right\rangle \\
& =\frac{3}{2} \sum \lambda_{k}^{-1}\left[\left(\boldsymbol{u}_{k}\right)_{i}^{2}-2\left(\boldsymbol{u}_{k}\right)_{i}\left(\boldsymbol{u}_{k}\right)_{j}+\left(\boldsymbol{u}_{k}\right)_{j}^{2}\right]
\end{aligned}
$$

There are two contributions to $\left\langle\Delta R_{i j}^{2}\right\rangle$. First, if residues $i$ and $j$ exhibit large fluctuations in space, the first and the third terms in Eq. (3) are large, reflecting the effects of mobility of each residue. The second is the correlation effect given by the second term in Eq. (3), which decreases the relative fluctuations if $i$ and $j$ move in phase (correlated), and increases if they are out of phase (anticorrelated).

For each of the the 15 ligand-receptor systems given in Table I, we formed the $\boldsymbol{\Gamma}$ matrix separately for ligand and receptor, each in the unbound state, and evaluated the eigenvalues and eigenvectors using Eq. (2). Then, we evaluated the modal components of $\left\langle\Delta R_{i j}^{2}\right\rangle$ using Eq. (3). We observe that for the ligand molecule, there exists resides that are strongly anticorrelated in the slowest mode with most of the remaining residues of the ligand.
Similarly, for the receptor molecule, there exists residues that are strongly coupled in the fastest mode with most of the remaining residues of the receptor. This coupling is in the form of a strong correlation with some of the residues and a strong anticorrelation with the remaining ones. We then show that these residues are either (i) previously identified by others as anchor residues, hotspots, or conserved residues, or (ii) are the residues in close proximity in the bound state. These results are general, with the exception of ASP99 and TYR33 shown in Table I with low conservation scores. All the predicted binding residue pairs across the interface are within the first coordination shell of $7 \AA$. In 8 out 15 cases, this distance is below $3.5 \AA$.

For illustrative purposes, we present our findings for a specific enzyme-inhibitor complex structure, 1BRC. In Fig. 1, the predictions on the unbound enzyme 1BRA (green) and the ligand 1AAP (blue) are presented on complex 1BRC. The anchor residues ARG15 and GLY37, shown in red, are strongly anticorrelated with most of the remaining residues in the slowest mode. Receptor residue ASP194 and GLY197, shown in blue, are identified from the fastest mode.

Anchor residues. - The main anchor residue mostly resides in the ligand; nevertheless, there are some cases (i.e., 1MEL.pdb in Table I) where the anchor is at the receptor. Figure 2(a) displays the correlation of the $i$ th residue with all other residues $j$ of the ligand of the complex structure 1BRC in the slowest mode of motion in its unbound state. Each column of points corresponding to a given residue $i$ represents the correlations $\left\langle\Delta R_{i j}^{2}\right\rangle$ of the $i$ th residue with all others. There are three peaks. The two peaks centered at ARG15 and GLY37, which are the anchor residues, display 


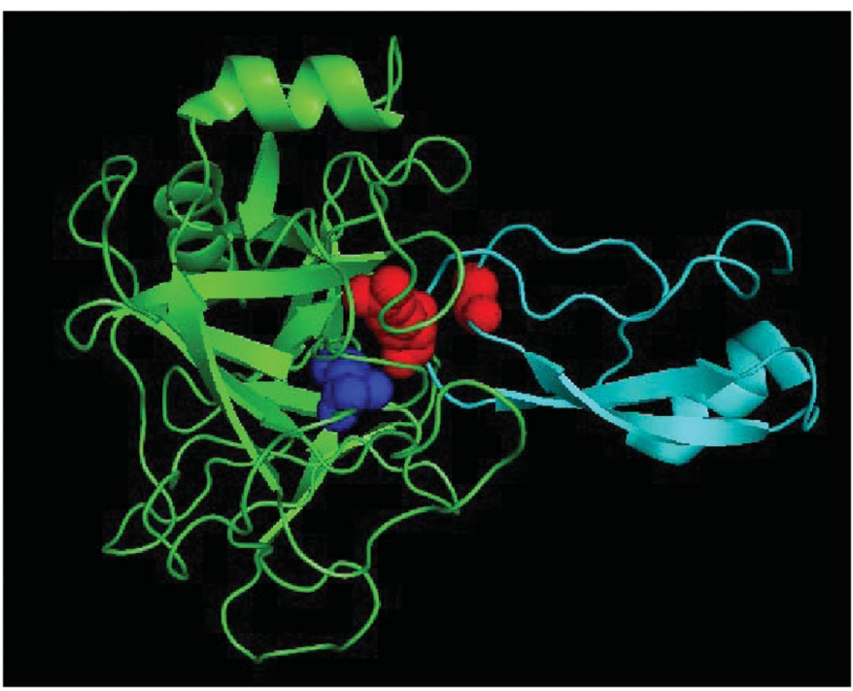

FIG. 1 (color). The enzyme (green)-ligand (blue) complex structure 1BRC. Binding residues are obtained from the model on unbound receptor and ligand, 1BRA, and 1AAP, respectively (see text for details).

the most negatively correlated fluctuations with the rest of the structure $\left(\left\langle\Delta \boldsymbol{R}_{i} \cdot \Delta \boldsymbol{R}_{j}\right\rangle<0\right)$ in addition to their high mobilites, $\left\langle\Delta R_{i}^{2}\right\rangle$ and $\left\langle\Delta R_{i}^{2}\right\rangle$. The peak centered at THR26 results from high mobility of this residue but is not significantly correlated with the rest of the ligand in this mode. THR26 is not associated with binding. In Fig. 2(b), correlations of $\left\langle\Delta R_{i 1}^{2}\right\rangle$ and $\left\langle\Delta R_{\text {in }}^{2}\right\rangle$ of residue $i$ with the first and last residues of the chain are presented. The anchor residues ARG15 and GLY37 exhibit anticorrelation with the tail residues. There is a significant symmetry: Arg 15 is anticorrelated equally with both ends of the chain. Same is true for Gly37. THR26 in this mode is not correlated with the tails although it has high mobility. Thus, although the temperature factor of a residue is a measure of mobility alone, it is not a measure for predicting binding residues.
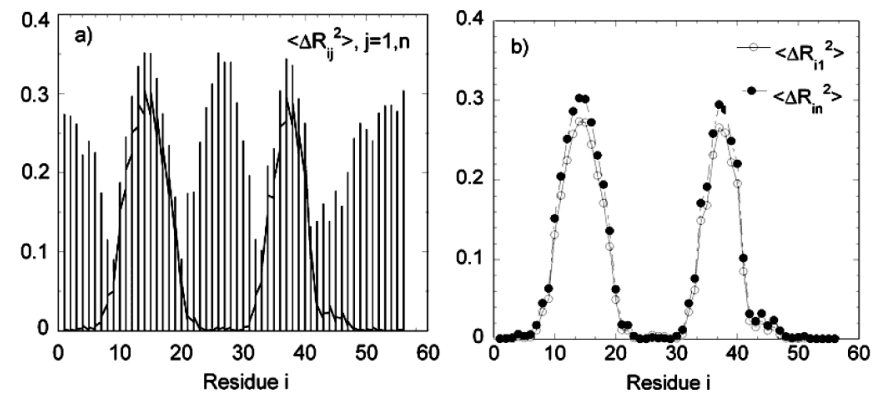

FIG. 2. Slowest mode relative fluctuations (a) $\left\langle\Delta R_{i j}^{2}\right\rangle$ of residue $i$ with all other residues, for the native unbound ligand of the enzyme-inhibitor 1BRC, 1AAP, indicated by the dots along a column corresponding to the residue $i$, (b) $\left\langle\Delta R_{i, 1}^{2}\right\rangle$ and $\left\langle\Delta R_{i, n}^{2}\right\rangle$ of residue $i$ with the first and the last ( $n$ th) residue of the ligand.
Anchoring groove residues. - Fluctuations $\left\langle\Delta R_{i j}^{2}\right\rangle$ in the fast modes of motion in Eq. (3) identify the residues in the unbound receptor structure that are highly correlated with the rest of the residues. These are spots on the unbound receptor surface that interact with the anchor of the ligand in the complex state. The results of the analysis for 15 cases are presented in Table I. For the case study of 1BRC, GNM calculations show that ASP194 and GLY197 (blue spheres in Fig. 1) are the residues in the unbound receptor, 1BRA, which displays the highest amplitude correlated fluctuations with the other residues of the receptor in the fastest mode. A parallel behavior is shown in Fig. 3(b), where an external manipulation picks ASP194 and couples it to the rest of the residues (see the discussion below). The correlation of ASP194 is stronger for some of the residues, which are also possibly associated with the binding.

Harmonic manipulation of ligand and receptor identifies the anchor and binding groove residues. - If an external harmonic force excites the $k$ th mode of the protein, then correlations of this mode with others will emerge. The total coupling, $C_{i j}(k)$, between the excited kth mode and all other modes for residues $i$ and $j$ by the GNM is given by [12]

$$
\begin{aligned}
C_{i j}(k) & =\sum_{m}\left\langle\Delta \boldsymbol{R}_{i}(k) \cdot \Delta \boldsymbol{R}_{j}(m)\right\rangle \\
& =\sum_{m} \frac{\lambda_{k} \lambda_{m}+\zeta^{2} \omega^{2}}{2\left(\lambda_{k}^{2}+\zeta^{2} \omega^{2}\right)\left(\lambda_{m}^{2}+\zeta^{2} \omega^{2}\right)} \boldsymbol{u}_{i}(k) \cdot \boldsymbol{u}_{j}(m)
\end{aligned}
$$

where $\zeta$ is the friction, and $\omega$ is the perturbing frequency, and $\zeta^{2} \omega^{2}$ acts as a scaling factor that comes from the solution of the Langevin equation [2] for the GNM. If only positive $\left\langle\Delta \boldsymbol{R}_{i}(k) \cdot \Delta \boldsymbol{R}_{j}(m)\right\rangle$ values are used in the summation in Eq. (4), then $C_{i j}(k)$ indicates correlation and vice versa. Exciting the ligand in the slowest mode $(k=1)$ does not excite all the residues but only the anchor residues ARG15 and GLY37, which in turn couple to all other residues, as shown in Fig. 3(a). Here, the abscissa denotes the residues that are excited in the slowest mode, and the ordinate denotes all the other residues to which the excited residues are coupled in highest number of modes.
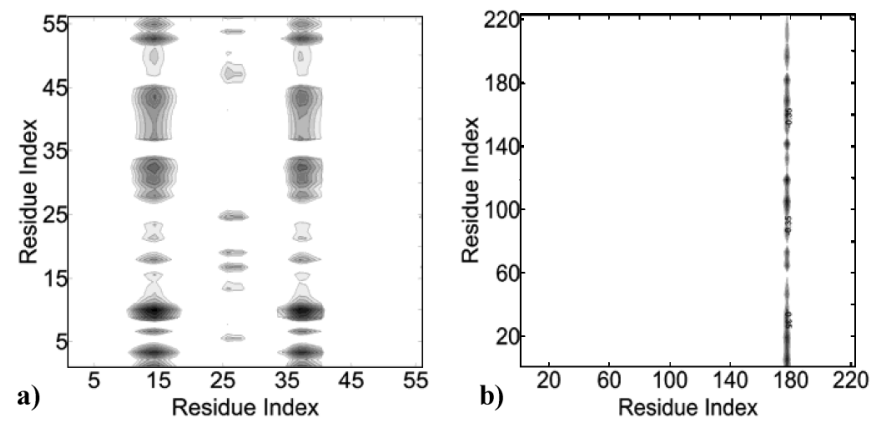

FIG. 3. Total correlation, $C_{i j}(k)$, for (a) the ligand (1AAP) with the slowest mode, and (b) for the receptor (1BRA) with the fastest mode. 
The contours denote the degree of coupling. The two vertical strips show the degree of correlations of ARG15 and GLY37 with the rest of the protein. For the receptor, shown in Fig. 3(b), only the site around residue ASP194 is excited in the fastest mode and is correlated with the rest of the protein as indicated by the vertical strip. This is the same residue identified by Eq. (3) in the preceding section. The abscissa values shown here should be augmented by 18 for correspondence to the $\mathrm{pdb}$ values.

Complex structure. - In the complex structure, the anchor residue of the ligand fluctuates in the same sense as the binding groove residues and the rest of the receptor residues, while it fluctuates in the negative sense with the rest of the ligand residues. Thus, the anchor residue hooks the ligand to the receptor. The negative cooperative motion of anchor residues with the rest of the ligand residues, and the high frequency vibrating behavior of anchoring groove residues, are imprinted in the unbound structure. These residues are more in buried regions, in cavities, of the interface in both unbound and bound states of the receptor and are identifiable in both states with the high frequency correlation analysis of the residues. Further, the anchor residues upon binding also appear in the high frequency dynamic mode of the complex structure by forming tight interactions with the anchoring groove residues. In the complex state, both anchor residues and the achoring groove residues should contribute to the binding stability with their tight interactions and could conveniently be referred to as "hotspot" residues of the complex structure.

Conclusion. - The anchor residues of the 15 benchmark proteins [1] are in good agreement with those obtained by extensive molecular dynamics simulations of Camacho et al. . The corresponding anchoring groove residues are those that are in the first coordination shell of the anchor residues.

The anchor residue is strongly anticorrelated with the rest of the residues of the ligand in the slow modes, which allows it to efficiently probe its environment. The anchor residues do not exhibit high conservation levels. However, their nearest neighbors are highly conserved. On the other hand, the binding grove residues of the receptors (i) are identified by the fastest modes; (ii) show a remarkable degree of conservation. That there is a relation between hotspot residues and structurally conserved residues has been shown previously $[7,9,13,14]$; (iii) are the residues that are most excited by external perturbation at the fast modes; and (iv) can transfer this perturbation to the rest of the protein because they are strongly coupled with the remaining residues of the receptor.

In the systems studied, the anchor lies on a convex surface, and the anchoring grove residues are on the concave surfaces of the receptor. This specific congruity al- lows the present model to identify the binding residues in terms of fastest and slowest modes only. The present analysis is also applied to homodimers and heterodimers with comparable sizes, where the concave and convex regions may be located on either or both of the binding surfaces of a pair $[5,15]$. The binding residue is the one that exhibits the highest correlation with the tails of the monomer, and may therefore be uniquely identified. Prediction now requires a larger number of modes depending on the size and the number of interactions that the protein might have. Similarly, the fastest few modes identify the binding residue if it is located in a grove in either one or both of the interfaces of the pairs investigated.

Preliminary studies show that the binding sites of flexible proteins that exhibit floppy binding, i.e., binding before or during folding [16], can also be identified with the present model.

T. H. acknowledges BU Research Grant No. 04HA502, State Planning Organization Grant No. 03K120250, EU No. FP6-2004-ACC-SSA-2: Project No. 517991.

[1] D. Rajamani, S. Thiel, and S. Vajda et al., Proc. Natl. Acad. Sci. U.S.A. 101, 11287 (2004).

[2] K. Falk, O. Rotzschke, and S. Stevanovic et al., Nature (London) 351, 290 (1991).

[3] W. DeLano, Curr. Opin. Struct. Biol. 12, 14 (2002).

[4] D. Reichmann, O. Rahat, and M. Cohen et al., Curr. Opin. Struct. Biol. 17, 67 (2007).

[5] I. M. A. Nooren and J. M. Thornton, J. Mol. Biol. 325, 991 (2003).

[6] I. Bahar, A. R. Atilgan, and B. Erman, Folding \& Design 2, 173 (1997).

[7] T. Haliloglu, I. Bahar, and B. Erman, Phys. Rev. Lett. 79, 3090 (1997).

[8] I. Bahar, A. R. Atilgan, and M.C. Demirel et al., Phys. Rev. Lett. 80, 2733 (1998).

[9] T. Haliloglu, O. Keskin, and M. Buyong et al., Biophys. J. 88 (3), 1552 (2005).

[10] R. Cheng, J. Mintseris, and J. Janin et al., Proteins: Struct. Funct. Genet. 52, 88 (2003).

[11] C. Berezin, F. Glaser, and Y. Rosenberg et al., Bioinformatics 20, 1322 (2004).

[12] A. Erkip and B. Erman, Polymer, 45, 641 (2004).

[13] B. Ma, T. Tal Elkayam, and H. Wolfson et al., Proc. Natl. Acad. Sci. U.S.A. 100, 5772 (2003).

[14] A. Ertekin, R. Nussinov, and T. Haliloglu, Protein Sci. 15, 2265 (2006).

[15] Genome Informatics 2003, edited by M. Gribskov, M. Kanehisa, S. Miyano, and T. Takagi (Universal Academy Press, Tokyo, 2003), Vol. 14, p. 238.

[16] Q. Lu, P. H. Lu, and J. Wang, Phys. Rev. Lett. 98, 128105 (2007). 